\title{
Nonlocal particles as strings
}

\author{
Tai-Chung Cheng ${ }^{1}$, Pei-Ming $\mathrm{Ho}^{2}$ and Tze-Kei Lee ${ }^{2}$ \\ ${ }^{1}$ Taipei Municipal Zhung-Lun High School, Taipei, Taiwan, Republic of China \\ ${ }^{2}$ Department of Physics and Center for Theoretical Sciences, National Taiwan University, Taipei, \\ Taiwan, Republic of China \\ E-mail: pmho@phys.ntu.edu.tw
}

Received 9 July 2008, in final form 19 November 2008

Published 6 January 2009

Online at stacks.iop.org/JPhysA/42/055202

\begin{abstract}
We find nonlocal particle theories with two-dimensional conformal symmetry, including examples equivalent to the bosonic open string and closed string. This work provides a new approach to construct solvable consistent backgrounds in string theory.
\end{abstract}

PACS numbers: $11.25 . \mathrm{Hf}, 11.25 .-\mathrm{w}$

\section{Introduction}

Many of the remarkable features of string theory are often attributed to the dimensionality of the string, since these features are not remotely shared by any known particle theory. For example, a necessity of the T-duality is the existence of winding modes, which is apparently absent in particle theory. Although it is also possible to take the viewpoint that string field theory is just a theory of infinitely many fields of particles, the spectrum of the fields should have an origin in a two-dimensional conformal field theory. The conformal symmetry is needed to ensure a consistent perturbation theory free of UV divergence and ghosts ${ }^{3}$. It is generally believed that the two-dimensional conformal symmetry is at the heart of many miracles of string theory.

The main idea behind this work is that the distinction between strings and particles is blurred when we consider particle actions that are nonlocal. Usually we assume that the equation of motion for a bosonic degree of freedom is a second-order differential equation, so that the phase space of a $D$-dimensional particle is $2 D$. In contrast, the string, viewed as a collection of infinitely many points, has a phase space of infinite dimensions. But for an equation of motion involving the $n$th derivatives, the phase space is $n D$ dimensional. When $n \rightarrow \infty$, it is possible that the phase space of a particle can be identified with that of a string. It was shown in [2] that the reparametrization symmetry of a particle's worldline approaches

3 Nevertheless, UV-finite, unitary field theories with infinitely many particles can also be constructed without reference to the conformal symmetry [1]. 
to the two-dimensional conformal symmetry in the $n \rightarrow \infty$ limit. This strongly suggests the equivalence between some nonlocal particle theories and string theories.

On the other hand, higher derivative theories have been known to suffer from many unphysical problems, such as non-unitarity, spectra unbounded from below, acausality, etc [3]. These problems are always present in any theory with higher derivatives of a finite order. Theories with infinite derivatives are hard to classify, and these problems may or may not be present. The higher derivative interactions in an effective theory should be treated in a perturbative approach [4]. Higher derivative interaction treated as a fundamental theory is unusual to most physicists.

In this paper, we find explicit (quadratic) nonlocal actions of particles which respect the two-dimensional conformal symmetry (section 2). The conformal symmetry is realized as the reparametrization symmetry of the particle worldline. Noether's theorem guarantees that the symmetry generators obey the Virasoro algebra (section 2.2).

We give many examples to illustrate the basic formulation, and suggest how a new dimension naturally arises on the worldline (to turn it into a worldsheet) in many cases. A generic nonlocal particle theory may not be unitary but it is easy to, and we give explicit rules for how to, construct examples preserving unitarity (section 3). Several unitary nonlocal particle theories are discussed in some detail. First we give a nonlocal particle theory which is equivalent to an open string in the trivial background (section 4). This equivalence guarantees that this nonlocal theory is well defined ${ }^{4}$. Next we give examples equivalent to open strings in nontrivial backgrounds (section 5). These nontrivial backgrounds can be described in terms of nonlocal interactions in the string worldsheet theory. Finally we define a nonlocal particle theory equivalent to a closed string in the flat background (section 6).

Our study reveals a new approach to construct consistent, solvable backgrounds in string theory. In general these new backgrounds correspond to higher derivative interactions on the string worldsheet, and are usually evaded in the traditional worldsheet approach to string theory. Our study also sheds some light on the conceptual question of whether our universe is made of particles or strings.

\section{Nonlocal actions with conformal symmetry}

\subsection{Canonical quantization of nonlocal particle action}

In order to have solvable equations of motion, we restrict ourselves to quadratic actions

$$
S=-\int \mathrm{d} t x(t) f\left(\partial_{t}\right) y(t)
$$

This action involves two scalar fields $x$ and $y$. The case of a single scalar field can be easily derived from this case by identifying $x(t)$ with $y(t)$.

Let us carry out the canonical quantization. The variation of this action is

$\delta S=\int \mathrm{d} t\left\{\delta x\left[f\left(\partial_{t}\right) y(t)\right]+\delta y(t)\left[f\left(-\partial_{t}\right) x(t)\right]+\left(x(t)\left[f\left(\partial_{t}\right) \delta y(t)\right]-\left[f\left(-\partial_{t}\right) x(t)\right] \delta y(t)\right)\right\}$,

where the last term in $(\cdots)$ is a total derivative. The equations of motion are

$$
f\left(-\partial_{t}\right) x(t)=0, \quad f\left(\partial_{t}\right) y(t)=0 .
$$

The most general solution of $x$ and $y$ are

$$
x(t)=\sum_{a} x_{a} \mathrm{e}^{-\mathrm{i} k_{a} t}, \quad y(t)=\sum_{a} y_{a} \mathrm{e}^{\mathrm{i} k_{a} t},
$$

4 The nonlocal particle theory for worldsheet ghosts is also found (section 4.3). 
where $\left\{k_{a}\right\}$ is the set of zeros of $f$ :

$$
\mathcal{Z}=\left\{k_{a}\right\}=\{k \mid f(\mathrm{i} k)=0\} .
$$

The total derivative in (2) is

$$
x(t)\left[f\left(\partial_{t}\right) \delta y(t)\right]-\left[f\left(-\partial_{t}\right) x(t)\right] \delta y(t)=\frac{\mathrm{d}}{\mathrm{d} t} \theta,
$$

where

$$
\theta=\bullet\left(\frac{1 \otimes f\left(\partial_{t}\right)-f\left(-\partial_{t}\right) \otimes 1}{1 \otimes \partial_{t}+\partial_{t} \otimes 1}\right)(x(t) \otimes \delta y(t)) .
$$

Here $\bullet$ is the symbol for multiplication, i.e. $\bullet(f \otimes g)=f g$. The quotient of the functions of derivative on the right-hand side of (7) is well defined as long as $f\left(\partial_{t}\right)$ admits a Taylor expansion

$$
f\left(\partial_{t}\right)=\sum_{n=0}^{\infty} f_{n} \partial_{t}^{n} .
$$

The symplectic two-form $\omega=\delta \theta$ is then

$$
\begin{aligned}
\omega & =\bullet\left(\frac{1 \otimes f\left(\partial_{t}\right)-f\left(-\partial_{t}\right) \otimes 1}{1 \otimes \partial_{t}+\partial_{t} \otimes 1}\right)(x(t) \otimes \delta y(t)) \\
& =\sum_{n=0}^{\infty} f_{n}\left\{\bullet \sum_{k=1}^{n}\left[\left(-\partial_{t}\right)^{k-1} \otimes \partial_{t}^{n-k}\right](\delta x(t) \otimes \delta y(t))\right\} .
\end{aligned}
$$

Assuming that all zeros of $f$ are simple (non-degenerate), we find

$$
\omega=\sum_{a} \dot{f}\left(\mathrm{i} k_{a}\right) \delta x_{a} \delta y_{a} .
$$

To derive this expression we note that (9) contains the factor

$$
\text { - }\left(\frac{1 \otimes f\left(\mathrm{i} k_{b}\right)-f\left(\mathrm{i} k_{a}\right) \otimes 1}{1 \otimes \mathrm{i} k_{b}-\mathrm{i} k_{a} \otimes 1}\right)
$$

when acting on the term $x_{a} \mathrm{e}^{-\mathrm{i} k_{a} t} \otimes y_{b} \mathrm{e}^{\mathrm{i} k_{b} t}$ in $x \otimes y$. Since $f\left(\mathrm{i} k_{a}\right)=f\left(\mathrm{i} k_{b}\right)=0$, this factor vanishes unless $k_{a}=k_{b}$. When $k_{a}=k_{b}$, it is obvious that this factor is supposed to be understood as the derivative of $f$, i.e., $\dot{f}\left(\mathrm{i} k_{a}\right)$. From the viewpoint of both the symplectic two-form and the Hamiltonian, a pair of variables $\left(x_{a}, y_{a}\right)$ is independent of another pair $\left(x_{b}, y_{b}\right)$ unless $k_{a}=k_{b}$.

If there are double poles at $k_{a}$, the general solution is of the form

$$
x=\left(x_{a}+p_{a} t\right) \mathrm{e}^{-\mathrm{i} k_{a} t}+\cdots, \quad y=\left(y_{a}+q_{a} t\right) \mathrm{e}^{\mathrm{i} k_{a} t}+\cdots,
$$

and the relevant part of the symplectic two-form will be

$$
\omega=\frac{1}{2} \ddot{f}\left(\mathrm{i} k_{a}\right)\left(\delta x_{a} \delta q_{a}+\delta y_{a} \delta p_{a}\right)+\cdots .
$$

This is easy to derive from (9). One can consider this as the limiting case of two nearby simples zeros $k_{a}, k_{b}$ with the difference approaching to zero, $\left(k_{a}-k_{b}\right) \rightarrow 0$. Similarly, for triple poles or poles of higher order degeneracy, it is straightforward to derive the symplectic two-form from (9) in the same way, although the theory would then have either a Hamiltonian unbounded from below or negative norm states. 


\subsection{Particle actions with $2 D$ conformal symmetry}

There are nonlocal particle actions with conformal symmetry, which is realized as the symmetry of worldline reparametrization

$$
x(t) \rightarrow x^{\prime}(t)=x(t+\delta t)
$$

For reparametrizations in the harmonic basis

$$
\delta t=\epsilon \mathrm{e}^{\mathrm{i} n t}+\text { c.c., } \quad \forall n \in \mathbb{Z},
$$

where $\epsilon \in \mathbb{C}$ and c.c. means complex conjugate, the infinitesimal transformation of $x$

$$
\delta x(t) \equiv x^{\prime}(t)-x(t)=\epsilon \mathrm{e}^{-\mathrm{i} n t} \dot{x}(t)+\text { c.c. }
$$

is generated by the differential operator

$$
V_{n}=\mathrm{e}^{-\mathrm{i} n t} \partial_{t}
$$

satisfying the classical Virasoro algebra

$$
\left[V_{m}, V_{n}\right]=(m-n) V_{m+n} .
$$

In the above we have restricted the values of the index $n$ to be integers so that the algebra generated by $V_{n}$ 's is no more than the Virasoro algebra. For $t$ compactified on a circle (this is of course not physical), we can always normalize $t$ so that $t \in[0,2 \pi)$, then $\left\{\mathrm{e}^{\mathrm{i} n t}\right\}_{n \in \mathbb{Z}}$ constitute a complete basis for functions of $t$ and the Virasoro algebra is equivalent to the full reparametrization symmetry of $t$. For the uncompactified time direction $t \in \mathbb{R}$, if we demand the most general reparametrization symmetry, $n$ can be any real number and the particle dynamics would have to be trivial. By restricting the values of $n$ to integers, we can still have many nontrivial models as shown below. Note that a length scale is introduced here corresponding to the shift $\Delta t$ in $t$ that will make all transformations in (17) trivial. We are using the unit system so that this length scale is normalized to $2 \pi$.

With these Virasoro generators imposed as constraints, the theory of the nonlocal particle has the gauge symmetry of conformal group, which is the defining feature of string theory.

The symmetry (17) implies that if $x(t)=\mathrm{e}^{-\mathrm{i} z t}$ is a solution to the equation of motion, then $x(t)=\mathrm{e}^{-\mathrm{i}(z+n) t}$ must also be a solution for any integer $n .^{5}$ As long as $f(\mathrm{i} k)$ satisfies the following property:

if $f\left(\mathrm{i} k_{a}\right)=0, \quad$ then $f\left(\mathrm{i}\left(k_{a}+n\right)\right)=0 \quad$ for all $n \in \mathbb{Z}$,

the action (1) has the conformal symmetry (17). The zeros of the function $f$ must come in sequences

$$
\mathcal{Z}=\left\{z_{i}+n ; n \in \mathbb{Z}\right\}
$$

The zeros $z_{i}$ constitute a minimal set of zeros such that (21) includes all the zeros of $f$.

The general solution of the equations of motion is

$$
x(t)=\sum_{k \in \mathcal{Z}} x_{k} \mathrm{e}^{-\mathrm{i} k t}, \quad y(t)=\sum_{k \in \mathcal{Z}} y_{k} \mathrm{e}^{\mathrm{i} k t} .
$$

Without loss of generality we can restrict $z_{i}$ 's to lie within the range $[0,1)$. If $f$ is symmetric, $f\left(-\partial_{t}\right)=f\left(\partial_{t}\right), z_{i}$ always comes in pairs $\left(z_{i},-z_{i}\right)$ except $z_{i}=0$ or $1 / 2$. The symplectic two-form (14) is now

$$
\omega=\sum_{k \in \mathcal{Z}} \dot{f}(\mathrm{i} k) \delta x_{k} \delta y_{k} .
$$

Here and below we assume that all zeros of $f$ are non-degenerate and real.

5 There is an exception when $z_{i} \in \mathbb{Z}$. Due to the derivative of $x$ in the transformation rule (17), $k=0$ does not have to be a zero of $f$. We will give examples on this in section 2.4.3. 
The conserved charges are $\theta$ with $\delta x, \delta y$ given by the symmetry transformation (17):

$$
Q_{n}=\sum_{k \in \mathcal{Z}} \mathrm{i}(k+n) \dot{f}(\mathrm{i} k) x_{k} y_{(k+n)} .
$$

According to (23), we define the creation, annihilation operators

$$
a_{-k}=(\dot{f}(\mathrm{i} k) k)^{1 / 2} x_{k}, \quad b_{k}=(\dot{f}(\mathrm{i} k) k)^{1 / 2} y_{k},
$$

so that the symplectic two-form is equivalent to

$$
\omega=\sum_{k \in \mathcal{Z}} \frac{\delta a_{-k} \delta b_{k}}{k} .
$$

If $k=\left(z_{i}+n\right)>0, a_{-k}$ is the creation operator and $b_{k}$ is the annihilation operator. If $k=\left(z_{i}+n\right)<0, a_{-k}$ is the annihilation operator and $b_{k}$ is the creation operator. In order for (25) to make sense, we assume here that

$$
\dot{f}(\mathrm{i} k) k>0
$$

at all zeros of $f$ because this is the condition that the Hamiltonian is bounded from below. A sequence of zeros $\left\{z_{i}+n\right\}$ is viewed as spacelike if (27) holds. If the opposite holds

$$
\dot{f}(\mathrm{i} k) k<0
$$

at a sequence of zeros, that sequence should be viewed as timelike, and the definition of $a_{k}, b_{k}$ in (25) should be modified by adding factors of $(-1)$ in the square roots. The contribution of a timelike sequence of zeros of $f$ to the Hamiltonian is bounded from above. For the theory to be consistent, we should demand that the Virasoro constraints be sufficient to eliminate unwanted states so that the spectrum of physical states is bounded from below. No-ghost theorem still needs to be proved.

The conserved charges are

$$
Q_{n}=\sum_{k \in \mathcal{Z}} B(n, k) a_{-k} b_{k+n},
$$

where

$$
B(n, k)=\left(\frac{\dot{f}(\mathrm{i} k)}{\dot{f}(\mathrm{i}(k+n))} \frac{k+n}{k}\right)^{1 / 2} .
$$

In particular, the Hamiltonian is

$$
Q_{0}=\sum_{k \in \mathcal{Z}} a_{-k} b_{k},
$$

which has a non-negative spectrum upon quantization after normal ordering. Using the property of $B(n, m)$

$$
B(n, \ell) B(m, n+\ell)=B(n+m, \ell),
$$

one can easily check that the Virasoro algebra is satisfied at the classical level (in Poisson brackets)

$$
\left(Q_{m}, Q_{n}\right)=-(m-n) Q_{m+n} .
$$

Note that the function $B(n, k)$ transforms under the similar transformation

$$
Q_{m} \rightarrow U Q_{m} U^{-1}, \quad U=\mathrm{e}^{\sum_{k} \lambda_{k} a_{-k} b_{k}}
$$

as

$$
B(n, k) \rightarrow \frac{\mathrm{e}^{k \lambda_{k}}}{\mathrm{e}^{(n+k) \lambda_{(n+k)}}} B(n, k) .
$$


Choosing

$$
\lambda_{k}=\frac{1}{2 k} \log [k / \dot{f}(\mathrm{i} k)]
$$

we get

$$
B(n, k) \rightarrow 1 .
$$

This means that the function $f\left(\partial_{t}\right)$ has no real physical significance, except when (36) is ill-defined, e.g. $\dot{f}(\mathrm{i} k)=0$ or $k=0$.

If $\dot{f}(\mathrm{i} k)=0, f$ has a degeneracy of zeros at $k$. This is assumed to be not the case in our derivation. In the above we have also assumed that $k \neq 0$ (see (26)). Hence, as far as we have considered, we can always set $B(n, k)=1$ without loss of generality.

To summarize, for the action (1), a nonlocal particle theory with conformal symmetry is characterized by its spectrum $\mathcal{Z}$. All possible $f\left(\partial_{t}\right)$ 's with the same spectrum are equivalent if $\dot{f}(\mathrm{i} k) k>0$ for all $k \in \mathcal{Z}$. After change of variables, the Virasoro algebra can always be realized in terms of the creation annihilation operators for excitation modes of this spectrum simply as

$$
Q_{n}=\sum_{k \in \mathbb{Z}} a_{-k} b_{k+n} .
$$

Compared with the spectrum of an open string, each sequence of zeros $\left\{z_{i}+n: n \in \mathbb{Z}\right\}$ is expected to correspond to one dimension in the target space. The sequences come in pairs $\left(z_{i},-z_{i}\right)$, except when $z_{i}=0$ or $z_{i}=1 / 2$ (so that $\pm z_{i}$ define the same sequence). In view of the spectrum of the sequences of zeros, we expect that $z_{i}=0$ corresponds to open strings with Neumann boundary conditions on both endpoints, and $z_{i}=1 / 2$ to open strings with the Neumann boundary condition on one endpoint and the Dirichlet boundary condition on the other endpoint. This will be shown more explicitly below, and we will discuss the generic case with $z_{i} \in(0,1 / 2)$ in section 4 .

Incidentally, we remark that the particle theory (1) does not exhaust all possible realization of the Virasoro algebra in terms of creation and annihilation operators. We give a counterexample in the appendix.

The formulae above can be easily modified to adapt to the case of a single field

$$
x=y \text {. }
$$

First, the function $f\left(\partial_{t}\right)$ should be symmetric $f\left(\partial_{t}\right)=f\left(-\partial_{t}\right)$. Thus the zeros of $f$ will come in pairs $(k,-k)$

$$
\mathcal{Z}=\{k \mid f(\mathrm{i} k)=0\}=\left\{ \pm z_{i}+n ; n \in \mathbb{Z}\right\},
$$

except when the zeros are on the fixed points $\mathbb{Z}$ or $\mathbb{Z}+1 / 2$. Without loss of generality we can assume that $z_{i} \in[0,1 / 2]$.

The general solution of $x$ is

$$
x=\frac{1}{\sqrt{2}} \sum_{i} \sum_{n \in \mathbb{Z}}\left(x_{\left(z_{i}+n\right)} \mathrm{e}^{-\mathrm{i}\left(z_{i}+n\right) t}+y_{\left(z_{i}+n\right)} \mathrm{e}^{\mathrm{i}\left(z_{i}+n\right) t}\right) .
$$

If there are zeros on the fixed points, we get in the general solution of $x$

$$
x_{0}+p t+\sum_{n \neq 0} x_{n} \mathrm{e}^{\mathrm{i} n t} \quad \text { and/or } \quad \sum_{n \in \mathbb{Z}} x_{n+1 / 2} \mathrm{e}^{\mathrm{i}(n+1 / 2) t} .
$$

The simplectic two-form $\omega$ is given by the same expressions (23) above. The conserved charges become

$$
Q_{n}=\sum_{k \in \mathcal{Z}}[(k+n) \dot{f}(\mathrm{i} k)+k \dot{f}(\mathrm{i}(k+n))] x_{-k} y_{k+n},
$$

assuming that the fixed points do not appear in $\mathcal{Z}$.

6 


\subsection{Quantization}

Let us consider Virasoro generators of the form

$$
L_{n}=\sum_{k \in \mathcal{Z}} B(n, k): a_{-k} b_{k+n}:
$$

where $a_{k}, b_{k}$ satisfy

$$
\left[a_{k}, b_{\ell}\right]=k \delta_{k+\ell}^{0} .
$$

This is just (29) with normal ordering. For $k>0, a_{k}, b_{k}$ are annihilation operators, and for $k<0, a_{k}, b_{k}$ are creation operators. Then we have

$$
\left[L_{m}, a_{k}\right]=-k B(m,-m-k) a_{m+k}, \quad\left[L_{m}, b_{k}\right]=-k B(m, k) b_{m+k} .
$$

From this it is straightforward to check that if $B$ satisfies the identity

$(k+m) B(m, k) B(n, k+m)-(k+n) B(n, k) B(m, k+n)=\frac{1}{2}(m-n) B(m+n, k)$,

the Virasoro algebra is guaranteed

$$
\left[L_{m}, L_{n}\right]=(m-n) L_{m+n}-\delta_{m+n}^{0} C(n),
$$

where

$$
C(n)=-\sum_{-n<k<0, k \in \mathcal{Z}} k(k+n) B(n, k) B(-n, k+n) .
$$

For $B(n, k)=1$, we get $C(n)=\frac{c}{12} n(n-1)(n+1)$ with the central charge $c=2$ for each sequence of zeros of $f$, as it should be for two scalar fields $x$ and $y$. For the generic expression of $B(30)$ in the nonlocal particle theory, we have $B(n, k) B(-n, k+n)=1$, and so

$$
C(n)=\sum_{m=1}^{n}(m-z)(n-m+z)=\frac{1}{6} n(n-1)(n+1)+n z(1-z)
$$

for the contribution of a sequence $k=z+m(m \in \mathbb{Z})$. We can absorb the last term into $L_{0}$ by shifting $L_{0}$ by the constant $z(1-z) / 2$. The central charge is therefore always $c=2$ for each sequence of zeros.

If there is only one scalar field, i.e., $x=y$, the contribution of each sequence of zeros to the central charge is 1 .

The contribution of a sequence of zeros of $f$ to the Casimir energy $a$ (ground-state energy of the Hamiltonian) can be computed as

$$
\begin{aligned}
a=\sum_{k}\left(a_{-k} b_{k}-\right. & \left.: a_{-k} b_{k}:\right)=\sum_{k<0}(-k)=\sum_{m=1}^{\infty}(m-z) \\
& =\frac{1}{24}-\frac{1}{8}(2 z-1)^{2}=-\frac{1}{12}+\frac{1}{2} z(1-z) .
\end{aligned}
$$

When $z=0$, this gives $a=-1 / 12$ as it should for two flat directions in the target space.

The $z$-dependent piece in $a$ suggests that we shift $L_{0}$ by the same number $z(1-z) / 2$ as it was suggested by $C(n)$ above. In terms of the shifted operator $L_{0}$, the contribution of a sequence of zeros of $f$ to the central charge and the zero-point energy are the same as two flat directions in the target space. Nevertheless, the conformal field theory defined by a generic $f$ can certainly be inequivalent to an open string in a flat background, because the spectrum $\left\{z_{i}+n\right\}$ and Virasoro constraints are different. 
Given the same ghost system as the open string (which will be described as ghost of a nonlocal particle below), we should select 26 sequences of zeros of $f$ so that the total central charge of the Virasoro algebra is 0 . The shifted $L_{0}$ should be defined as

$$
L_{0}=\sum_{i}\left[\sum_{k_{i}=z_{i}+\mathbb{Z}}: a_{-k_{i}} b_{k_{i}}:+\frac{1}{2} z_{i}\left(1-z_{i}\right)\right] \text {, }
$$

and the Virasoro constraints should be

$$
L_{m}-a \delta_{m}^{0}=0, \quad m \geqslant 0,
$$

where $a=\frac{S-T}{24}$. Here $S$ and $T$ are the number of sequences of zeros of $f$ for target space coordinates which are spacelike or timelike. Since $S+T=26$ in order to cancel the Virasoro anomaly of the ghost, here we want $T=1$ and $S=25$, and so $a=1$.

\subsection{Classification}

In the case $x=y$,

$$
S=-\frac{1}{2 \pi} \int \mathrm{d} t x f\left(\partial_{t}\right) x
$$

Without loss of generality we can assume that the function $f\left(\partial_{t}\right)$ is even

$$
f\left(-\partial_{t}\right)=f\left(\partial_{t}\right) .
$$

The equation of motion is

$$
f\left(\partial_{t}\right) x=0 .
$$

If the transformation (17) is a symmetry, given a solution $x$ of the equation of motion, $\mathrm{e}^{\mathrm{i} n t} \dot{x}$ must also be a solution. That is, we need

$$
f\left(\partial_{t}+\mathrm{i} n\right) \dot{x}=0
$$

whenever $x$ satisfies (56).

We will consider three classes of $f$ with conformal symmetry. These are the cases when the nonlocal particles would be naturally described as a string because, as we will see below, the symplectic two-form and conserved charges can be naturally expressed as integrals of a fictitious variable. The mathematical identity that will help us to do this is

$$
A(t+a) B(t)-A(t) B(t-a)=\frac{\mathrm{d}}{\mathrm{d} t}\left[\int_{0}^{a} \mathrm{~d} \beta A(t+\beta) B(t+\beta-a)\right] .
$$

To show how this formula leads to a fictitious dimension on the nonlocal particle, we will carry out the canonical quantization all over again, although the results above can be applied to all the examples of this section.

2.4.1. First class. For the first class, $f$ is a periodic or anti-periodic function

$$
f\left(\partial_{t}+\mathrm{i}\right)= \pm f\left(\partial_{t}\right) .
$$

Given (59), the conformal symmetry condition (57) is equivalent to

$$
( \pm)^{n} \partial_{t}\left[f\left(\partial_{t}\right) x^{\mu}\right]=0,
$$

which holds whenever the equation of motion is satisfied. Since we have assumed that $f$ is an even function, its Fourier expansion is

$$
f\left(\partial_{t}\right)=\sum_{n}^{\prime} f_{n} \mathrm{e}^{n \pi \partial_{t}}, \quad f_{-n}=f_{n},
$$


where $\sum_{n}{ }^{\prime}$ denotes a sum over either even or odd numbers, depending on whether $f$ is periodic or anti-periodic.

Let us start with one spacetime dimension for the first class. Variation of the action is

$$
\begin{aligned}
\delta S=\frac{1}{2 \pi} \int \mathrm{d} t & \left\{\delta x(t) f\left(\partial_{t}\right) x(t)+x(t) \sum_{n}^{\prime} f_{n} \delta x(t+n \pi)\right\} \\
= & \frac{1}{2 \pi} \int \mathrm{d} t\left\{\delta x(t) f\left(\partial_{t}\right) x(t)+\sum_{n}^{\prime} f_{n}\{x(t-n \pi) \delta x(t)\right. \\
& \left.\left.+\frac{\mathrm{d}}{\mathrm{d} t}\left[\int_{0}^{n \pi} \mathrm{d} \beta x(t+\beta-n \pi) \delta x(t+\beta)\right]\right\}\right\} \\
= & \frac{1}{2 \pi} \int \mathrm{d} t\left\{2 \delta x(t) f\left(\partial_{t}\right) x(t)+\frac{\mathrm{d}}{\mathrm{d} t} \sum_{n}^{\prime} f_{n}\right. \\
& \left.\times\left[\int_{0}^{n \pi} \mathrm{d} \beta x(t+\beta-n \pi) \delta x(t+\beta)\right]\right\} .
\end{aligned}
$$

The symplectic two-form is the differential of the total derivative part of $-\delta S$

$$
\omega=\frac{1}{2 \pi} \sum_{n}^{\prime} f_{n}\left[\int_{0}^{n \pi} \mathrm{d} \beta \delta x(t+\beta-n \pi) \delta x(t+\beta)\right] .
$$

Noether's theorem gives us the conserved charges for the symmetry transformations (17)

$$
\delta t=\epsilon \mathrm{e}^{-\mathrm{i} m t}
$$

as

$$
Q_{m} \frac{1}{2 \pi} \sum_{n}^{\prime} f_{n}\left[\int_{0}^{n \pi} \mathrm{d} \beta \mathrm{e}^{-\mathrm{i} m(t+\beta)} \dot{x}(t+\beta) x(t+\beta-n \pi)\right],
$$

where the equation of motion is used to simplify the expression.

2.4.2. Second class. The second class of actions with conformal symmetry is given by (54) with

$$
f\left(\partial_{t}\right)=g\left(\partial_{t}\right) \partial_{t}
$$

where $g\left(\partial_{t}\right)$ is an odd periodic or anti-periodic function

$$
g\left(\partial_{t}+\mathrm{i}\right)= \pm g\left(\partial_{t}\right), \quad g\left(-\partial_{t}\right)=-g\left(\partial_{t}\right) .
$$

The condition for conformal symmetry (57) is then

$$
( \pm)^{n}\left(\partial_{t}+\mathrm{i} n\right)\left[f\left(\partial_{t}\right) x^{\mu}\right]=0
$$

whenever $x$ satisfies the equation of motion. This is obviously valid. The Fourier expansion of $g$ is

$$
g\left(\partial_{t}\right)=\sum_{n \geqslant 0}^{\prime} g_{n} \mathrm{e}^{n \pi \partial_{t}}, \quad g_{-n}=-g_{n}
$$

Again the sum is only over either even or odd numbers. 
Variation of the action is

$$
\begin{aligned}
\delta S=-\frac{1}{2 \pi} \int \mathrm{d} t & \left\{\delta x(t) f\left(\partial_{t}\right) x(t)+x(t) \sum_{n}^{\prime} g_{n} \delta \dot{x}(t+n \pi)\right\} \\
= & -\frac{1}{2 \pi} \int \mathrm{d} t\left\{\delta x(t) f\left(\partial_{t}\right) x(t)+\sum_{n}^{\prime} g_{n}\{x(t-n \pi) \delta \dot{x}(t)\right. \\
& \left.\left.+\frac{\mathrm{d}}{\mathrm{d} t}\left[\int_{0}^{n \pi} d \beta x(t+\beta-n \pi) \delta \dot{x}(t+\beta)\right]\right\}\right\} \\
= & -\frac{1}{2 \pi} \int \mathrm{d} t\left\{2 \delta x(t) f\left(\partial_{t}\right) x(t)+\frac{\mathrm{d}}{\mathrm{d} t} \sum_{n}^{\prime} g_{n}[-x(t+n \pi) \delta x(t)\right. \\
& \left.\left.+\int_{0}^{n \pi} \mathrm{d} \beta x(t+\beta-n \pi) \delta \dot{x}(t+\beta)\right]\right\} .
\end{aligned}
$$

The symplectic two-form is thus

$\omega=\frac{1}{2 \pi} \sum_{n}^{\prime} g_{n}\left[-\delta x(t+n \pi) \delta x(t)+\int_{0}^{n \pi} \mathrm{d} \beta \delta x(t+\beta-n \pi) \delta \dot{x}(t+\beta)\right]$.

The conserved charges for the transformation (64) are then

$$
\begin{aligned}
& Q_{m}=\frac{1}{2 \pi} \sum_{n}^{\prime} g_{n}\left[-x(t+n \pi) \mathrm{e}^{-\mathrm{i} m t} \dot{x}(t)+\int_{0}^{n \pi} \mathrm{d} \beta x(t+\beta-n \pi) \partial_{\beta}\left(\mathrm{e}^{-\mathrm{i} m(t+\beta)} \dot{x}(t+\beta)\right)\right] \\
&= \frac{1}{2 \pi} \sum_{n}^{\prime} g_{n}\left[-\int_{0}^{n \pi} \mathrm{d} \beta \mathrm{e}^{-\mathrm{i} m(t+\beta)} \dot{x}(t+\beta) \dot{x}(t+\beta-n \pi)\right. \\
&\left.-(x(t-n \pi)+x(t+n \pi)) \mathrm{e}^{\mathrm{i} m t} \dot{x}(t)+x(t) \mathrm{e}^{-\mathrm{i} m(t+n \pi)} \dot{x}(t+n \pi)\right] \\
&= \frac{-1}{2 \pi} \sum_{n}^{\prime} g_{n}\left[\int_{0}^{n \pi} \mathrm{d} \beta \mathrm{e}^{-\mathrm{i} m(t+\beta)} \dot{x}(t+\beta) \dot{x}(t+\beta-n \pi)\right. \\
&\left.+\mathrm{e}^{-\mathrm{i} m t}(x(t-n \pi)+x(t+n \pi)) \dot{x}(t)\right] \\
&= \frac{-1}{2 \pi} \sum_{n}^{\prime} g_{n}\left[\int_{0}^{n \pi} \mathrm{d} \beta \mathrm{e}^{-\mathrm{i} m(t+\beta)} \dot{x}(t+\beta) \dot{x}(t+\beta-n \pi)\right] .
\end{aligned}
$$

Again the equation of motion is used to simplify the expression.

Since these are the conserved charges derived from the symmetry (64), the Poisson brackets among the charges $Q_{m}$ are given by the classical Virasoro algebra

$$
\left(Q_{m}, Q_{n}\right)=(m-n) Q_{m+n} .
$$

Examples of this class of models include the nonlocal particle theory equivalent to an open string with Neumann boundary conditions on both endpoints (see section 4.1), or the Neumann boundary condition on one endpoint but the Dirichlet boundary condition on the other endpoint (4.2).

2.4.3. Third class. The third class is the exceptional case mentioned above. It has

$$
f\left(\partial_{t}\right)=\frac{h\left(\partial_{t}\right)}{\partial_{t}},
$$

10 
where $h\left(\partial_{t}\right)$ is an odd periodic function

$$
h\left(\partial_{t}+\mathrm{i}\right)=( \pm) h\left(\partial_{t}\right), \quad h\left(-\partial_{t}\right)=-h\left(\partial_{t}\right) .
$$

The Fourier expansion of $h$ is the same as (69). If $h$ has only a simple zero at $k \in \mathbb{Z}, f$ has no zero at $k=0$, but has a zero at all other integers. Thus the sequence of zeros is not complete. Nevertheless, the condition (57) is now

$$
( \pm)^{n}\left(\partial_{t}+\mathrm{i} n\right)^{-1} \partial_{t}\left[h\left(\partial_{t}\right) x(t)\right]=0 .
$$

This holds because the equation of motion $f\left(\partial_{t}\right) x=0$ implies that $h\left(\partial_{t}\right) x=0$.

Since the inverse operator of $\partial_{t}$ is not always well defined, we will only consider the cases when $h(0)=0$, e.g. $h\left(\partial_{t}\right)=\tanh \left(\pi \partial_{t}\right)$. For the general solution

$$
x(t)=\sum_{n \neq 0} x_{n} \mathrm{e}^{\mathrm{i} n t}
$$

it is straightforward to repeat the same derivation above to get

$$
\omega=C \sum_{n \neq 0} \frac{\delta x_{-n} \delta x_{n}}{\mathrm{i} n},
$$

where $C=\dot{h}(\mathrm{i})$, and

$$
Q_{m}=C \sum_{n \neq 0} \frac{m+n}{n} x_{-n} x_{m+n} .
$$

In this case it is not so easy to use the formula (58) to express $\omega$ and $Q_{m}$ as an integral, because the inverse of $\partial_{t}$ is not necessarily well defined.

An explicit example is the nonlocal particle equivalent to an open string with Dirichlet boundary conditions on both endpoints (see comments at the end of section 4.2).

\subsection{More than one dimensions}

One can easily extend the nonlocal particle theory in one dimensions to $D$-dimensional spacetime, replacing (54) by

$$
S=-\frac{1}{2 \pi} \int \mathrm{d} t x^{\mu} f\left(\partial_{t}\right) x_{\mu}
$$

This theory has Lorentz symmetry (but not necessarily translational symmetry). If we do not care about Lorentz symmetry, there can be more general nonlocal action with conformal symmetry

$$
S=\frac{1}{2 \pi} \int \mathrm{d} t x^{\mu} f_{\mu \nu}\left(\partial_{t}\right) x^{\nu},
$$

where $f_{\mu \nu}$ satisfies

$$
f_{\mu \nu}\left(-\partial_{t}\right)=f_{v \mu}\left(\partial_{t}\right), \quad \mu, v=0, \ldots, d .
$$

The $N$ th class $(N=-1,0,1)$ of action with conformal symmetry has

$$
f_{\mu \nu}\left(\partial_{t}\right)=g_{\mu \nu}\left(\partial_{t}\right) \partial_{t}^{N},
$$

where $g_{\mu \nu}\left(\partial_{t}\right)$ satisfies

$$
g_{\mu \nu}\left(\partial_{t}+\mathrm{i}\right)=\Lambda_{\mu}^{\lambda} g_{\lambda \nu}\left(\partial_{t}\right), \quad g_{\mu \nu}\left(-\partial_{t}\right)=(-1)^{N} g_{v \mu}\left(\partial_{t}\right),
$$

and $\Lambda_{\mu}^{\lambda}$ is an $S O(1, d)$ matrix. 


\section{Unitarity}

In the above we have only considered the mathematical formulation of nonlocal particles, without worrying about whether they make physical sense or not. As a simple example of how things can go wrong, we consider the case with

$$
g\left(\partial_{t}\right)=\sinh \left(\pi \partial_{t}\right) \quad\left(g_{1}=-g_{-1}=1 / 2, g_{n \neq 1}=0\right)
$$

for an action of the second class. The equation of motion is

$$
\dot{x}(t+\pi)=\dot{x}(t-\pi)
$$

and the general solution is

$$
x(t)=x_{0}+y_{0} t+\sum_{n \neq 0} \frac{y_{n}}{\mathrm{i} n} \mathrm{e}^{\mathrm{i} n t} .
$$

According to (71), the symplectic two-form is

$$
\omega=\delta x_{0} \delta y_{0}+\sum_{n \neq 0} \frac{(-1)^{n}}{2 \mathrm{i} n} \delta y_{n} \delta y_{-n} .
$$

The inverse of the symplectic two-form is the Poisson bracket

$$
\left(x_{0}, y_{0}\right)=1, \quad\left(y_{n}, y_{-n}\right)=(-1)^{n} 2 \mathrm{i} n .
$$

The Poisson bracket for $x$ is thus

$$
\left(x(t), \dot{x}\left(t^{\prime}+\pi\right)\right)=-1+\sum_{n \in \mathbb{Z}} 2 \mathrm{e}^{\mathrm{i} n\left(t-t^{\prime}\right)}=-1+4 \pi \delta\left(t-t^{\prime}\right) .
$$

Upon quantization, equation (89) is equivalent to

$$
\left[y_{m}, y_{n}\right]=(-1)^{m} 2 m \delta_{m+n}^{0} .
$$

The Virasoro generators are

$$
\begin{aligned}
Q_{m} & =\frac{1}{4 \pi}\left[-\int_{0}^{\pi} \dot{x}(t+\beta-\pi) \mathrm{e}^{-\mathrm{i} m(t+\beta)} \dot{x}(t+\beta) \mathrm{d} \beta+\int_{0}^{-\pi} \dot{x}(t+\beta+\pi) \mathrm{e}^{-\mathrm{i} m(t+\beta)} \dot{x}(t+\beta) \mathrm{d} \beta\right] \\
& =\frac{-1}{4 \pi}\left[\int_{-\pi}^{\pi}\left(y_{0}+\sum_{n} y_{n} \mathrm{e}^{\mathrm{i} n(t+\beta-\pi)}\right) \mathrm{e}^{-\mathrm{i} m(t+\beta)}\left(y_{0}+\sum_{n^{\prime}} y_{n^{\prime}} \mathrm{e}^{\mathrm{i} n^{\prime}(t+\beta)}\right)\right] \\
& =\frac{-1}{2} \sum_{n}(-1)^{n} y_{n} y_{m-n},
\end{aligned}
$$

which vanish for odd $m$. The Hamiltonian is

$$
H=Q_{0}=\frac{-1}{2} \sum_{n}(-1)^{n} y_{-n} y_{n} .
$$

Apparently the operators $y_{m}$ and $Q_{m}$ are reminiscent of $\alpha_{m}$ and $L_{-m}$ in the open string theory. The only difference is the extra factors of $(-1)^{n}$ 's, and this difference is crucial. Due to these $(-1)^{n}$ factors, either the Hilbert space contains negative-norm states, or the Hamiltonian is unbounded from below. From (93), we see that the Hamiltonian is unbounded from below due to oscillation modes with even $n$. One might want to avoid this pathology at the quantum level by defining the vacuum to be annihilated by $y_{-n}$ for even $n>0$, so that states created by $y_{n}$ for even $n$ has positive energy. For example, for $m>0$, the state $y_{2 m}|0\rangle$ has positive energy

$$
H y_{2 m}|0\rangle=-y_{2 m}\left(-2 m+y_{2 m} y_{-2 m}\right)|0\rangle=2 m y_{2 m}|0\rangle .
$$


(The odd modes will then obey the usual convention that $y_{2 m+1}|0\rangle=0$ for $m \geqslant 0$.) But then this state is of negative norm

$$
\left\langle 0\left|y_{-2 m} y_{2 m}\right| 0\right\rangle=-2 m<0 \text {. }
$$

In fact, it is not necessary to go through the detailed computation to tell whether an action suffers the problem of negative-norm states or the problem of Hamiltonians unbounded from below. For an ordinary free scalar theory in $D$ dimensions, the Fourier transformation of two-point correlator is given by

$$
\langle\psi(p) \psi(q)\rangle=\delta^{(D)}(p-q) \frac{\mathrm{i}}{p^{2}-m^{2}+\mathrm{i} \varepsilon} .
$$

Now if the propagator has multiple poles, the correlator near the $i$ th pole is given by

$$
\langle\psi(p) \psi(q)\rangle \simeq \delta^{(D)}(p-q) \frac{\mathrm{i} C_{i}}{p^{2}-m_{i}^{2}+\mathrm{i} \varepsilon},
$$

where $C_{i}$ is the residue of the $i$ th pole. The sign of this quantity when $p$ and $q$ are on-shell (which is the same as the sign of $C_{i}$ because $\varepsilon>0$ ) is correlated with the sign of the norm of the single particle state with momentum $p$. Therefore the requirement of unitarity is equivalent to demanding the residues of all poles of the propagator, which is $1 / f(\mathrm{i} k)$ for our case, to be of the same sign. Otherwise, there will either be negative-norm states or the Hamiltonian will be unbounded from below.

In the example above, $f(\mathrm{i} k)=-k \sin (\pi k)$ and the residues of poles of the propagator $1 / f(\mathrm{i} k)$ have alternating signs. Hence half of the oscillation modes have negative energy while the other half have positive energy.

In the following we will demand that the theory be unitary (no negative norm states) and that the classical Hamiltonian be positive definite. (Tachyons can appear after including the quantum correction due to Casimir effect, yet the quantum spectrum will still be bounded from below.) In other words, we will demand that the residues of poles of $1 / f(\mathrm{i} k)$ be all positive. When $k$ is close to a pole of $1 / f(\mathrm{i} k)$, we have

$$
1 / f(\mathrm{i} k) \simeq \frac{1}{f^{\prime}\left(\mathrm{i} k_{0}\right)\left(k-k_{0}\right)},
$$

and thus the residue of the pole at $k_{0}$ is just $1 / f^{\prime}\left(\mathrm{i} k_{0}\right)$. This means that we shall demand that the slope of $f(\mathrm{i} k)$ at all zeros of $f(\mathrm{i} k)$ be positive.

\section{Open string as nonlocal particle}

\subsection{The Neumann boundary condition}

The requirement of unitarity implies that $g\left(\partial_{t}\right)$ must be periodic rather than anti-periodic, because the latter implies that half of the poles have negative residues. We also want $g\left(-\partial_{t}\right)=-g\left(\partial_{t}\right)$ (see (67)) to hold. A simple modification of our previous example that would meet our needs is

$$
g\left(\partial_{t}\right)=\tanh \left(\pi \partial_{t}\right)=\frac{\sinh \left(\pi \partial_{t}\right)}{\cosh \left(\pi \partial_{t}\right)} .
$$

The same action was considered by Kato [5] long time ago as a particle action equivalent to the bosonic open string.

Let us now derive this action from open string theory by integrating out the bulk degrees of freedom on the worldsheet, and show that this action is just the effective action for the boundary coordinates. 
For a quadratic action, the effect of integrating out a variable is the same as plugging a classical solution into the action. Thus, to integrate out $X(\tau, \sigma)$ in the bulk of the string, we only need its solution to the equation of motion for given boundary value $X(\tau, 0)=X_{0}(\tau)$. The general solution to the equation of motion

$$
\left(\partial_{\tau}^{2}-\partial_{\sigma}^{2}\right) X(\tau, \sigma)=0
$$

with the Neumann boundary condition $\partial_{\sigma} X=0$ at $\sigma=\pi$ is

$$
X(\tau, \sigma)=\int \mathrm{d} k \mathrm{e}^{\mathrm{i} k \tau} \cos (k(\sigma-\pi)) \tilde{x}(k),
$$

where $\tilde{x}(k)$ is determined by $X_{0}$

$$
\tilde{x}(k)=\frac{1}{2 \pi \cos (k \pi)} \int \mathrm{d} \tau \mathrm{e}^{-\mathrm{i} k \tau} X_{0}(\tau) .
$$

Plugging (101), (102) into the action, we get

$$
\begin{gathered}
S=\frac{1}{2 \pi} \int \mathrm{d} \tau \int_{0}^{\pi} \mathrm{d} \sigma\left(\left(\partial^{\tau} X\right)^{2}-\left(\partial_{\sigma} X\right)^{2}\right)=\frac{1}{2 \pi} \int \mathrm{d} \tau\left[X \partial_{\sigma} X\right]_{\sigma=0} \\
=-\frac{1}{2 \pi} \int \mathrm{d} \tau \mathrm{d} \tau^{\prime} X_{0}(\tau) G\left(\tau-\tau^{\prime}\right) X_{0}\left(\tau^{\prime}\right),
\end{gathered}
$$

where the kernal is

$$
G(\tau)=\frac{1}{2 \pi} \int \mathrm{d} k k \frac{\sin (k \pi)}{\cos (k \pi)} \mathrm{e}^{\mathrm{i} k \tau} .
$$

It is easy to check that this is equivalent to the action (54) with the function $f\left(\partial_{t}\right)=g\left(\partial_{t}\right) \partial_{t}$ where $g\left(\partial_{t}\right)$ is given by (99).

Since open string vertex operators only live on the worldsheet boundary, in principle we can turn an open string theory in any open string background to a nonlocal particle theory.

Note that not every different choice of $g\left(\partial_{t}\right)$ defines a different theory. The choice (99) is physically equivalent to any other choice of the form

$$
g\left(\partial_{t}\right)=g_{0}\left(\partial_{t}\right) \sinh \left(\pi \partial_{t}\right)
$$

as long as $g_{0}$ satisfies the following criteria:

- $g_{0}(\mathrm{i} k)$ has no zeros (so that the solution to the equation of motion is still (87)).

- $g_{0}(\mathrm{i} k)$ is anti-periodic (so that $g(\mathrm{i} k)$ is periodic).

- $g_{0}(\mathrm{i} k)=g_{0}(-\mathrm{i} k)$ (so that $g(\mathrm{i} k)$ is an odd function).

- $(-1)^{n} g_{0}(\mathrm{i} n \pi)>0$ (so that $g^{\prime}(\mathrm{i} k)$ is always positive at all zeros of $g(\mathrm{i} k$ ), which are $k=n \pi)$.

These properties are all we need for the derivation below.

The symplectic two-form (71) is

$$
\omega=C\left[2 \delta x_{0} \delta y_{0}+\sum_{n \neq 0} \frac{1}{\mathrm{i} n} \delta y_{n} \delta y_{-n}\right]
$$

where

$$
C \equiv \sum_{n}^{\prime} \frac{n}{2} g_{n}=\frac{1}{2 \pi} g^{\prime}(0) .
$$

Canonical quantization therefore gives

$$
\left[y_{m}, y_{n}\right]=m C^{-1} \delta_{m+n}^{0}, \quad\left[x_{0}, y_{0}\right]=\frac{1}{\mathrm{i} C} .
$$


According to (72), the Virasoro generators are

$$
Q_{m}=C \sum_{n \in \mathbb{Z}} y_{-m-n} y_{n}
$$

Up to overall constant factors of $C$ which can be absorbed by scaling the coordinate $x$, these equations are the same as the open string with the following identification (assuming that we scaled $C$ to $C=1$ ):

$$
x_{0}=x, \quad y_{0}=p, \quad y_{m}=\alpha_{m}, \quad Q_{m}=2 L_{-m} .
$$

\subsection{The Dirichlet boundary condition}

For an open string with one endpoint (at $\sigma=\pi$ ) obeying the Dirichlet boundary condition, we can also integrate out the bulk of the string to obtain the effective action for the other endpoint coordinate at $\sigma=0$

$$
X(\tau, \pi)=0, \quad X(\tau, 0)=X_{0}(\tau) .
$$

The general solution of the equation of motion (100) with the Dirichlet boundary condition is

$$
X(\tau, \sigma)=\int \mathrm{d} k \mathrm{e}^{\mathrm{i} k \tau} \sin (k(\pi-\sigma)) \tilde{x}(k) .
$$

Therefore,

$$
\tilde{x}(k)=\frac{1}{2 \pi \sin (k \pi)} \int \mathrm{d} \tau \mathrm{e}^{-\mathrm{i} k \tau} X_{0}(\tau)
$$

and we can plug it into the worldsheet action to get

$$
S=-\frac{1}{2 \pi} \int \mathrm{d} \tau \mathrm{d} \tau^{\prime} X_{0}(\tau) G\left(\tau-\tau^{\prime}\right) X_{0}\left(\tau^{\prime}\right),
$$

where

$$
G(\tau)=\frac{1}{2 \pi} \int \mathrm{d} k k \frac{\cos (k \pi)}{\sin (k \pi)} \mathrm{e}^{\mathrm{i} k(\tau)} .
$$

This corresponds to

$$
g\left(\partial_{t}\right)=\frac{\cosh \left(\pi \partial_{t}\right)}{\sinh \left(\pi \partial_{t}\right)}
$$

for our particle action (54) with $f\left(\partial_{t}\right)=g\left(\partial_{t}\right) \partial_{t}$.

The general solution to the equation of motion for the nonlocal particle is

$$
x(t)=\sum_{n \in \mathbb{Z}} x_{n+1 / 2} \mathrm{e}^{\mathrm{i}(n+1 / 2) t} .
$$

It follows that the symplectic two-form is

$$
\omega=\frac{1}{2} \sum_{n \in \mathbb{Z}} \frac{\delta y_{n+1 / 2} \delta y_{-n-1 / 2}}{n+1 / 2},
$$

and the conserved charges are

$$
Q_{m}=\frac{1}{2} \sum_{n \in \mathbb{Z}} \delta y_{n+1 / 2} \delta y_{m-n-1 / 2} .
$$

One might wonder what happens to the open string with Dirichlet boundary conditions on both endpoints? In this case we cannot apply the same trick used above. But we can construct the equivalent nonlocal particle theory directly from the spectrum of the open string, which is $\mathbb{Z}-\{0\}$. This spectrum suggests a model of the third class with $h\left(\partial_{t}\right)=\tanh \left(\pi \partial_{t}\right)$. It is obvious that this nonlocal particle theory is equivalent to the open string theory with Dirichlet boundary conditions on both endpoints. 


\subsection{Ghosts}

To fully match with the open string theory, we also need to rewrite the ghosts living on the string worldsheet as a particle theory. The action for the two-dimensional $b c$ conformal field theory should be

$$
S_{g}=\frac{\mathrm{i}}{2 \pi} \int \mathrm{d} t b(t) g\left(\partial_{t}\right) c(t)
$$

where $b$ and $c$ are anticommuting fields. The action is invariant under the symmetry transformation

$\delta b(t)=\epsilon \mathrm{e}^{\mathrm{i} n t}(\dot{b}(t)+\mathrm{i} n \lambda b(t)), \quad \delta c(t)=\epsilon \mathrm{e}^{\mathrm{i} n t}(\dot{c}(t)+\mathrm{i} n(1-\lambda) c(t))$,

where $(\lambda, 1-\lambda)$ are the conformal weights of $b$ and $c$.

It is straightforward to derive the symplectic two-form

$$
\omega=\frac{\mathrm{i}}{2 \pi} \sum_{n}^{\prime} g_{n} \int_{0}^{n \pi} \mathrm{d} \beta \delta b(t+\beta-n \pi) \delta c(t+\beta),
$$

and the conserved charges

$Q_{m}=\frac{\mathrm{i}}{2 \pi} \sum_{n}^{\prime} g_{n} \int_{0}^{n \pi} \mathrm{d} \beta \mathrm{e}^{\mathrm{i} m(t+\beta)} b(t+\beta-n \pi)[\dot{c}(t+\beta)+\mathrm{i} m(1-\lambda) c(t+\beta)]$.

Substituting the general solutions of the equation of motion

$$
b=\sum_{n=-\infty}^{\infty} b_{n} \mathrm{e}^{-\mathrm{i} n t}, \quad c=\sum_{n=-\infty}^{\infty} c_{n} \mathrm{e}^{-\mathrm{i} n t},
$$

into the symplectic two-form (122) and conserved charges (123), one finds

$$
\begin{aligned}
& \omega=\mathrm{i} C \sum_{n=-\infty}^{\infty} b_{n} c_{-n}, \\
& Q_{m}=C \sum_{n=-\infty}^{\infty}[m(\lambda-1)-n] b_{m+n} c_{n} .
\end{aligned}
$$

We should take $\lambda=2$ for the ghosts of open string theory. The 26-dimensional bononic open string theory can thus be viewed as a 26-dimensional theory of nonlocal particles with the action (54) of the second class (66) with $g\left(\partial_{t}\right)$ given by (99), plus a ghost with the action (120).

Now that we have all the algebraic elements of open string theory, we can construct vertex operators and define scattering amplitudes in exactly the same way we define perturbative open string theory. The vertex operator is defined as a local operator $V$ with conformal dimension 1

$$
\left[Q_{m}, V\right]=\mathrm{e}^{\mathrm{i} m t}\left(-\mathrm{i} \frac{\mathrm{d}}{\mathrm{d} t}+m\right) V
$$

For example, the tachyon vertex should be given by

$$
\begin{aligned}
V_{0}(k, t) & =: \mathrm{e}^{\mathrm{i} k \cdot X(t)}: \\
& =\exp \left(k \cdot \sum_{n=1}^{\infty} \frac{y_{-n}}{n} \mathrm{e}^{\mathrm{i} n t}\right) \mathrm{e}^{\mathrm{i} k \cdot x(t)} \exp \left(-k \cdot \sum_{n=1}^{\infty} \frac{y_{-n}}{n} \mathrm{e}^{-\mathrm{i} n t}\right),
\end{aligned}
$$

where

$$
k^{2}=2, \quad X^{\mu}(t)=x_{0}^{\mu}+y_{0}^{\mu} t+\sum_{n \neq 0} \frac{y_{n}^{\mu}}{\mathrm{i} n} \mathrm{e}^{\mathrm{i} n t}, \quad x^{\mu}(t)=x_{0}^{\mu}+y_{0}^{\mu} t .
$$


Obviously all the scattering amplitudes are exactly the same as in open string theory at the tree level. The path integral approach to loop diagrams based on Riemann surfaces of different topology is obscured. However we can still take the operator approach [6] to construct the loop amplitudes. Anyway, there is a unique way to define loop diagrams without breaking conformal symmetry.

Fermionic fields can also be introduced on the worldline in a similar way as the ghosts. It should be straightforward to define nonlocal particles equivalent to superstrings. The worldsheet theory of a superstring is equivalent to the juxtaposition of free bosons, free fermions and ghosts, all of which we know how to describe in terms of nonlocal particles. Realizing that the oscillation modes on a string can be directly matched with those on a nonlocal particle, to derive the supersymmetry transformation rule for the nonlocal particle theory, one can simply copy the transformation rules for the oscillation modes on a superstring, and reinterpret them as the rules for the oscillation modes on a nonlocal particle.

Before closing this section, we also comment that, while the notion of a two-dimensional metric is absent in the particle theory, it seems impossible to talk about anything more than what is in the conformal gauge, e.g. the Weyl symmetry. On the other hand, since we have introduced a fictitious coordinate $\beta$ in expressions like (72), and thus the two-dimensional nature of the nonlocal particle is not totally invisible, it may be possible to introduce auxiliary fields to 'covariantize' these expressions, such that these auxiliary fields correspond to the two-dimensional metric on a string before gauge fixing. At that time we can start addressing questions concerning Weyl symmetry, etc.

\section{Open string in nontrivial backgrounds}

In the above we have shown that a certain choice of the function $f\left(\partial_{t}\right)$ in the nonlocal particle action (54) leads to a theory equivalent to the bosonic open string in flat space with the Neumann (or Dirichlet) boundary condition. A different choice of $f\left(\partial_{t}\right)$ would then lead to a theory equivalent to the bosonic open string in flat space with a nontrivial boundary interaction, corresponding to a nontrivial open string background, i.e., a nontrivial D-brane configuration. We will continue to focus on quadratic actions only.

Let us consider an explicit example where the action is of second class with

$$
g\left(\partial_{t}\right)=\frac{\sinh \left(\pi \partial_{t}+\mathrm{i} \theta\right) \sinh \left(\pi \partial_{t}-\mathrm{i} \theta\right)}{\sinh \left(2 \pi \partial_{t}\right)}
$$

The general solution to the equation of motion is

$$
x(t)=\sum_{n \in \mathbb{Z}} \frac{y_{n}^{+}}{\mathrm{i}(n-\theta / \pi)} \mathrm{e}^{\mathrm{i}(n-\theta / \pi) t}+\sum_{n \in \mathbb{Z}} \frac{y_{n}^{-}}{\mathrm{i}(n+\theta / \pi)} \mathrm{e}^{\mathrm{i}(n+\theta / \pi) t} .
$$

The symplectic two-form is

$$
\omega=\sum_{n \in \mathbb{Z}} \frac{\delta y_{n}^{+} \delta y_{-n}^{-}}{\mathrm{i}(n-\theta / \pi)} .
$$

The conserved charges are

$$
Q_{l}=-\sum_{n \in \mathbb{Z}} y_{n}^{+} y_{-n-l}^{-}
$$

The expression of the symplectic two-form suggests that we decompose $x(t)$ in another way

$$
x(t)=\tilde{x}^{+}(t)+\tilde{x}^{-}(t)
$$


where

$$
\tilde{x}^{ \pm}=\sum_{n} \frac{\tilde{y}_{n}^{ \pm}}{\mathrm{i}(n \mp \operatorname{sgn}(n) \theta / \pi)} \mathrm{e}^{\mathrm{i}(n \mp \operatorname{sgn}(n) \theta / \pi) t},
$$

where the function $\operatorname{sgn}(n)$ is defined by $\operatorname{sgn}(n) \equiv n /|n|$ for $n \neq 0$ and $\operatorname{sgn}(0)=1$, and

$$
\tilde{y}_{n}^{ \pm}=y_{n}^{ \pm} \quad(n \geqslant 0), \quad \tilde{y}_{n}^{ \pm}=y_{n}^{\mp} \quad(n<0) .
$$

Upon quantization, we get

$$
\left[\tilde{y}_{0}^{+}, \tilde{y}_{0}^{-}\right]=-\frac{\theta}{\pi}
$$

and

$$
\left[\tilde{y}_{n}^{+}, \tilde{y}_{-n}^{+}\right]=n-\theta / \pi, \quad\left[\tilde{y}_{n}^{-}, \tilde{y}_{-n}^{-}\right]=n+\theta / \pi \quad \text { for } \quad n>0 .
$$

Let us make a digression here and consider the limit $\theta \rightarrow 0$ and change of variables

$$
x_{0} \equiv \frac{\tilde{y}_{0}^{+}-\tilde{y}_{0}^{-}}{-2 \mathrm{i} \theta / \pi}, \quad y_{0}^{\prime} \equiv \tilde{y}_{0}^{+}+\tilde{y}_{0}^{-} .
$$

Commutation relations are now

$$
\left[x_{0}, y_{0}^{\prime}\right]=-\frac{\mathrm{i}}{2 C(\theta)}, \quad\left[\tilde{y}_{n}^{+}, \tilde{y}_{-n}^{+}\right]=\frac{n}{2 C(\theta)}, \quad\left[\tilde{y}_{n}^{-}, \tilde{y}_{-n}^{-}\right]=\frac{n}{2 C(\theta)} \text {. }
$$

Interestingly, the operators have a one-to-one correspondence with those of the closed string

$$
x_{0}=x, \quad y_{0}^{\prime}=p, \quad \tilde{y}_{m}^{+}=\alpha_{m}, \quad \tilde{y}_{m}^{-}=\tilde{\alpha}_{m},
$$

where the notation is such that the closed string target space variable is

$$
X(\tau, \sigma)=x+p \tau+\frac{\mathrm{i}}{\sqrt{2}} \sum_{n \neq 0}\left[\frac{\alpha_{m}}{m} \mathrm{e}^{\mathrm{i} m(\tau+\sigma)}+\frac{\tilde{\alpha}_{m}}{m} \mathrm{e}^{\mathrm{i} m(\tau-\sigma)}\right] .
$$

It appears that the closed string may be viewed as a special limit of a more general nonlocal particle model. However this observation has to be taken with a grain of salt, because the closed string theory has two commuting copies of Virasoro algebra for the left-moving and right-moving modes, but there is only one copy of Virasoro algebra for this particle theory. We will see how to generalize our ansatz for nonlocal particles to incorporate closed strings.

This nonlocal particle theory is actually equivalent to an open string theory with the usual bulk Lagrangian in the flat space and a nonlocal boundary interaction

$$
S=\frac{1}{2 \pi} \int \mathrm{d} \tau \mathrm{d} \sigma \partial X \bar{\partial} X+\left.\int \mathrm{d} \tau X h\left(\partial_{\tau}\right) \dot{X}\right|_{\sigma=0},
$$

where $h\left(\partial_{\tau}\right)$ is the difference between (130) and (99)

$$
h\left(\partial_{\tau}\right)=\frac{\sinh \left(\pi \partial_{t}+\mathrm{i} \theta\right) \sinh \left(\pi \partial_{t}-\mathrm{i} \theta\right)}{\sinh \left(2 \pi \partial_{t}\right)}-\frac{\sinh \left(\pi \partial_{t}\right)}{\cosh \left(\pi \partial_{t}\right)} .
$$

This should be obvious from our derivation in section 4.1. We have thus found an exact, marginal (nonlocal) deformation of the bosonic open string theory. Nonlocal interactions in string theory have been studied in the past in different contexts [7].

However, in section 2.2 we commented that the only thing that matters physically in the quadratic nonlocal particle action is the spectrum. The description above is not giving the essential properties of the theory in the most transparent way as it depends on too much detail of $f$. Instead, we can try to find a string worldsheet theory with the same spectrum. Given the two sequences of zeros $\{n \pm \theta / \pi\}$, it is natural to construct a worldsheet scalar field

$$
Z(\tau, \sigma)=\sum_{n \in \mathbb{Z}}\left[\alpha_{n} \mathrm{e}^{\mathrm{i}(n+\theta / \pi)(\tau+\sigma)}+\beta_{n} \mathrm{e}^{-\mathrm{i}(n+\theta / \pi)(\tau-\sigma)}\right],
$$


which gives the same general solution as (131)

$$
Z_{0}(\tau)=\sum_{n \in \mathbb{Z}}\left[\alpha_{n} \mathrm{e}^{\mathrm{i}(n+\theta / \pi) \tau}+\beta_{n} \mathrm{e}^{-\mathrm{i}(n+\theta / \pi) \tau}\right]
$$

at $\sigma=0$, and it satisfies the twisted periodic boundary condition

$$
Z(\tau, \sigma+2 \pi)=\mathrm{e}^{\mathrm{i} 2 \theta} Z(\tau, \sigma) .
$$

This is a boundary condition consistent with the standard worldsheet action in flat space, that is, it satisfies the condition

$$
\left.\left(\delta Z \partial_{\sigma} Z^{*}+\delta Z^{*} \partial_{\sigma} Z\right)\right|_{\sigma=0} ^{\sigma=2 \pi}=0
$$

necessary to guarantee that a solution of the equation of motion

$$
\partial \bar{\partial} Z=0
$$

extremizes the action.

Note that the twisted periodic boundary condition is not a typical open string boundary condition, which should not have any nonlocal correlation between the two endpoints of the string. This is, for example, not the same as the boundary conditions for an open string stretched between two D-branes separated by an angle $\theta$ in the complex plane of $Z$, because the values of $Z$ at $\sigma=0,2 \pi$ are not fixed. This is certainly not a closed string, either, since the string is not closed. This is a generalization of both open string and closed string, where the distinction between them is blurred.

\section{Closed string as nonlocal particle}

For the open string, we can interpret the nonlocal particle as the boundary of the open string. The equivalence between open string theory and particle theory is thus understandable. However, for the closed string, vertex operators can be inserted at any point on the worldsheet. No point on the closed string is special. It is hard to imagine how the closed string can also be equivalent to a nonlocal particle. Remarkably, we will show here that closed strings can also be described as nonlocal particles.

Roughly speaking, the degrees of freedom and its symmetry in a closed string is the same as two copies of those of an open string, but with the zero modes of the two open strings identified. Thus it is natural to consider the particle action

$S=\frac{1}{2 \pi} \int \mathrm{d} t\left[x(t) f\left(\partial_{t}\right) x(t)+y(t) f\left(\partial_{t}\right) y(t)+\lambda\left(\sinh \left(\pi \partial_{t}\right)(x(t)-y(t))\right)\right]$,

where

$$
f\left(\partial_{t}\right)=g\left(\partial_{t}\right) \partial_{t}, \quad g\left(\partial_{t}\right)=\tanh \left(\pi \partial_{t}\right)
$$

If the last term is absent in the action, we just have two copies of open string (with the Neumann boundary condition). We shall identify them with the left-moving and right-moving modes on the closed string. There are also two copies of the conformal symmetry and its Virasoro algebra generators for $x$ and $y$ independently. The last term in (150) involves a Lagrange multiplier $\lambda$. Note that since $\lambda$ is a constant variable, the last term is a total derivative. Hence the equations of motion for $x$ and $y$, as well as the symplectic two-form and conserved charge $Q_{m}$ are not modified. But it imposes the constraint

$$
\int \mathrm{d} t\left(\sinh \left(\pi \partial_{t}\right)(x(t)-y(t))\right)=0 .
$$


Plugging into solutions of $x$ and $y$

$$
x=x_{0}+p_{0} t+\sum_{n} x_{n} \mathrm{e}^{\mathrm{i} n t}, \quad y=y_{0}+q_{0} t+\sum_{n} y_{n} \mathrm{e}^{\mathrm{i} n t},
$$

the constraint implies that

$$
p_{0}=q_{0} .
$$

According to the symplectic two-form

$$
\omega=x_{0} p_{0}+y_{0} q_{0}+\cdots=\left(x_{0}+y_{0}\right) p_{0}+\cdots,
$$

this implies that the conjugate variable of $p_{0}$ is now $\left(x_{0}+y_{0}\right)$. The other linear combination $\left(x_{0}-y_{0}\right)$ is a non-dynamical constant which can be dismissed. As we have achieved the identification of the zero modes, the closed string is thus also described as a nonlocal particle theory.

\section{Discussion}

\subsection{Summary of nonlocal particle theory}

All physical properties of a nonlocal particle theory for two fields $x$ and $y$ with conformal symmetry are encoded in the spectrum, i.e., the zeros of $f$

$$
\mathcal{Z}=\left\{z_{i}+n: n \in \mathbb{Z}\right\}
$$

with each sequence specified by a number $z_{i} \in[0,1)$. Here we assume that $\dot{f}(\mathrm{i} k) k$ is of the same sign at all zeros of $f$, otherwise the theory is not unitary.

If $x=y, f$ is symmetric and a zero at $k$ implies a zero at $(-k)$. The data characterizing the conformal theory of a single scalar field $x$ are again just the zeros of $f$

$$
\mathcal{Z}=\left\{z_{i}+m,-z_{i}-m: m \in \mathbb{Z} \mid f\left(z_{i}\right)=0\right\}
$$

specified by a set of numbers $z_{i}$. Each $z_{i}$ specifies two sequences of zeros of $f$ unless $z_{i}=0$ or $z_{i}=1 / 2$. Other properties of $f$ are irrelevant. If there are two functions $f_{1}\left(\partial_{t}\right)$ and $f_{2}\left(\partial_{t}\right)$ with the same set of zeros and they differ from each other only by a function $\Delta f$ which vanishes at all the zeros of $f_{1}$ and $f_{2}$, the two theories defined by $f_{1}$ and $f_{2}$ are physically equivalent. For example, if the zeros of $f$ are at $\{z+n, n \in \mathbb{Z}\}$, then

$$
f(\mathrm{i} k)+a \sin ^{2}(\pi(k-z))
$$

is equivalent to $f(\mathrm{i} k)$.

\subsection{Nontrivial string backgrounds}

As it is easy to write down nonlocal particle actions which are equivalent to an open string in flat space with nonlocal interactions on the boundary (i.e., those with a spectrum of $z \neq 0$ or $1 / 2$ ), it will be very interesting to characterize the D-brane configurations corresponding to these nonlocal boundary interactions. It will also be interesting to study Witten's cubic string field theory [8] for these open string backgrounds.

We have demonstrated that by considering particle theory with higher derivatives, it is possible to obtain not only open but also closed bosonic string theory. Using this method, one can easily find new consistent backgrounds of string theory. Since solvable string backgrounds used to be rare, it will be very helpful to investigate string theory in these backgrounds for our understanding on the issue of background (in)dependence of string theory. 


\subsection{Boundary string field theory}

From the viewpoint of boundary string field theory, we are considering a class of solvable backgrounds with nonlocal interactions on the boundary. This kind of backgrounds was considered by Li and Witten [9]. It will be interesting to see if the nonlocal particle theories with conformal symmetry are solutions minimizing the boundary string field theory action.

In the boundary string field theory, the coefficients in the boundary interactions (coefficients in the function $f\left(\partial_{t}\right)$ ) correspond to spacetime fields. The fact that apart from the location of zeros, most details of $f$ are physically irrelevant indicates a large gauge symmetry in the boundary string field theory.

\subsection{Compactification and other backgrounds}

While we claimed that we have constructed a closed string theory out of nonlocal particles, an immediate question is whether we can consider compactification of space and find winding modes in the particle theory. This is a question that we will focus on in the near future. For the time being let us comment that the winding mode degrees of freedom can be easily found at the self-dual radius. If we compactify the target spaces of both $x$ and $y$ at the self-dual radius, both $p_{0}$ and $q_{0}$ are quantized. The linear combination $p_{0}+q_{0}$ corresponds to the momentum mode and $p_{0}-q_{0}$ to the winding mode. Earlier we imposed the constraint $p_{0}=q_{0}$ by a Lagrange multiplier $\lambda$. We can just drop the Lagrange multiplier to recover the winding modes in the particle theory. Once we can construct the string field theory at the self-dual radius, it should be possible to find configurations corresponding to compactifications at other values of the radius via a study of the associated Higgs mechanism.

On the other hand, it might not be possible to present all possible backgrounds of string theory as a nonlocal particle theory. The conformal field theory in the appendix with Virasoro generators defined by (29) with $B$ defined by (A.2) is such an example. The value of the nonlocal particle presentation of the string theory lies in its simplicity and abundance of new backgrounds.

\subsection{Relation to rigid strings}

There was an attempt [10] to describe the QCD string by the so-called 'rigid string' which has a worldsheet action involving higher derivative interactions. However there are only finite derivatives, and its high-temperature partition function was shown to have a wrong sign [11]. A natural question is whether it is possible to construct actions with infinite derivative (nonlocal) terms to reproduce the correct high energy limit of a QCD flux tube [12], and whether such strings can be described as a nonlocal particle.

Furthermore, while the rigid string can be viewed as a truncated description of a membrane with one direction wrapped on a compactified dimension [13], later it was shown that a 'rigid string' can also be viewed as derived from a string in a similar fashion [14]. The nonlocal string which is precisely equivalent to a string can thus be viewed as a completion of the approximation of a string by a 'rigid particle', which has only finite higher derivative terms.

Another topic that we will leave for future study is to find a nonlocal string/particle theory which is equivalent to a membrane. Since the quantum membrane theory is much less understood than strings, this equivalence may provide useful help for us to better understand the $M$ theory. 


\section{Acknowledgments}

The author thanks Chuan-Tsung Chan, Kazuyuki Furuuchi, Hiroyuki Hata, Takeo Inami, Nobuyuki Ishibashi, James Liu, Satchitananda Naik, Shunsuke Teraguchi, Wen-Yu Wen and Syoji Zeze for helpful discussions. This work is supported in part by the National Science Council, and the National Center for Theoretical Sciences (NSC 94-2119-M-002-001), Taiwan, Republic of China and the Center for Theoretical Physics at the National Taiwan University.

\section{Appendix. A non-particle example of conformal symmetry}

For generators of the form (29), without assuming (30), the Virasoro algebra (33) is satisfied if

$(k+m) B(m, k) B(n, k+m)-(k+n) B(n, k) B(m, k+n)=\frac{1}{2}(m-n) B(m+n, k)$.

This condition has a nontrivial solution

$$
B(n, k)=1+\frac{u+v n}{k}
$$

for constant parameters $u, v$. This can never be realized in a nonlocal particle theory because it does not satisfy (32). In order for $Q_{0}$ to have a non-negative spectrum, we would like

$$
B(0, k)=1+\frac{u}{k}
$$

to be positive-definite for all $k \in \mathcal{Z}$ (40). For $0<z_{i}<1$, this is possible if

$$
0<u<1-z_{i} \quad \text { or } \quad-z_{i}<u<0 .
$$

Upon quantization, the conserved charges are

$$
L_{n}=\sum_{k \in \mathcal{Z}} B(n, k): a_{-k} b_{k+n}:
$$

the Virasoro algebra is

$$
\left[L_{m}, L_{n}\right]=(m-n) L_{m+n}-\delta_{m+n}^{0} C(n),
$$

where

$$
\begin{aligned}
C(n)=- & \sum_{-n<k<0, k \in \mathcal{Z}} k(k+n) B(n, k) B(-n, k+n) \\
& =\frac{1}{6} n(n+1)(n-1)+n(z+u)(1-z-u) .
\end{aligned}
$$

This corresponds to $c=2$, and suggests a shift of $L_{0}$ by $(z+u)(1-z-u) / 2$. The central charge is 1 .

\section{References}

[1] Ho P M and Tian Y Y 2005 UV-finite scalar field theory with unitarity J. High Energy Phys. JHEP01(2005)026 (arXiv:hep-th/0410248)

[2] Ho P M 2003 Virasoro algebra for particles with higher derivative interactions Phys. Lett. B 558238 (arXiv: hep-th/0208182)

[3] Eliezer D A and Woodard R P 1989 The problem of nonlocality in string theory Nucl. Phys. B 325389

[4] Cheng T C, Ho P M and Yeh M C 2002 Perturbative approach to higher derivative and nonlocal theories Nucl. Phys. B 625151 (arXiv:hep-th/0111160)

Cheng T C, Ho P M and Yeh M C 2002 Perturbative approach to higher derivative theories with fermions Phys. Rev. D 66085015 (arXiv:hep-th/0206077) 
[5] Kato M 1990 Particle theories with minimum observable length and open string theory Phys. Lett. B 24543

[6] Green M B, Schwarz J H and Witten E Superstring Theory vol I (Cambridge: Cambridge University Press)

[7] Aharony O, Berkooz M and Silverstein E 2001 Multiple-trace operators and non-local string theories J. High Energy Phys. JHEP08(2001)006 (arXiv:hep-th/0105309)

Aharony O, Berkooz M and Silverstein E 2002 Non-local string theories on $\operatorname{AdS}(3) \times S^{3}$ and stable nonsupersymmetric backgrounds Phys. Rev. D 65106007 (arXiv:hep-th/0112178)

Barbon J L F and Hoyos C 2004 AdS/CFT, multitrace deformations and new instabilities of nonlocal string theories J. High Energy Phys. JHEP01(2004)049 (arXiv:hep-th/0311274)

[8] Witten E 1986 Noncommutative geometry and string field theory Nucl. Phys. B 268253

[9] Li K and Witten E 1993 Role of short distance behavior in off-shell open string field theory Phys. Rev. D 48853 (arXiv:hep-th/9303067)

[10] Polyakov A M 1986 Fine structure of strings Nucl. Phys. B 268406

Kleinert H 1986 The membrane properties of condensing strings Phys. Lett. B 174335

[11] Polchinski J and Yang Z 1992 High temperature partition function of the rigid string Phys. Rev. D 463667 (arXiv:hep-th/9205043)

[12] Polchinski J 1992 High temperature limit of the confining phase Phys. Rev. Lett. 68 1267 (arXiv: hep-th/9109007)

[13] Lindstrom U 1989 Deriving polyakov's rigid string from a membrane Phys. Lett. B 218315

[14] Pavsic M 1990 On The consistent derivation of rigid particles from strings Class. Quantum Grav. 7 L187 